\title{
Testicular and thyroid function as survival predictors in the elderly patient candidate to surgery
}

\author{
Antonio Aversa1, Andrea Fabbri2 \\ 1 Department Experimental and Clinical Medicine, University of Catanzaro "Magna Graecia" \\ 2 Unit of Endocrinology and Metabolic diseases, Department of Systems Medicine, University Tor Vergata, \\ Rome, Italy
}

\begin{abstract}
The relationship between testosterone deficiency (TD) syndrome and surgical resilience has a great impact in the modern approach to male elderly patients. There is good evidence that low levels of $\mathrm{T}$ are a strong marker for cardiovascular risk; also, TD is frequently associated with increased cardiovascular and all-cause mortality especially in cardiac older frail men. Screening for low T should be mandatory in high risk groups candidate to surgery including those with diabetes, metabolic syndrome and obesity, even though benefits from T-treatment on survival rates are unclear.

The low-T3 syndrome, named non-thyroidal illness (NTI) that occurs during critical illness refers to a syndrome with different faces in both sexes. The acute stress or critical illness-induced alterations within the thyroid axis occur in the first days of critical illness i.e. post-surgery and are brought about at least in part by the concomitant macronutrient deficit. The NTI that occurs in prolonged critically ill patients or in post-surgical resilience patients who continue to be dependent on intensive medical care for weeks or months, may have an impact on surgical outcomes because of frequent occurrence of cardiac arrhythmias. Future directions should better routinely investigate circulating thyroid hormones in population at risk before surgery after excluding iatrogenic drug interferences, and investigate the effect of possible treatments on survival rates after surgery.
\end{abstract}

Corresponding author: Prof. Antonio Aversa, Dipartimento Medicina Sperimentale e Clinica, Università di Catanzaro Magna Graecia, Viale Europa - Località Germaneto, 88100 Catanzaro, Italy. E-mail: aversa@unicz.it

Key words: Testosterone; male hypogonadism; thyroid hormone; mortality; resilience.

Received for publication: 1 November 2016

Accepted for publication: 9 November 2016

(C) Copyright A. Aversa and A. Fabbri, 2017

Tipografia PI-ME Editrice, Italy

Monaldi Archives for Chest Disease 2017; 87:841

doi: 10.4081/monaldi.2017.841

This article is distributed under the terms of the Creative Commons Attribution Noncommercial License (by-nc 4.0) which permits any noncommercial use, distribution, and reproduction in any medium, provided the original author(s) and source are credited.

\section{Background on testosterone}

The European Male Ageing Study (EMAS) reported a prevalence of testosterone deficiency (TD) at $5.1 \%$ in a non-obese male population of 70-79, whereas studies on populations with Type-2 diabetes report TD prevalence rates of $40 \%$ and of $15-30 \%$ in men presenting with erectile dysfunction. Accordingly, the presence of hypogonadism in men with cardiovascular disease (CVD) is about two times higher than observed in the general population and is associated with increase of the most known cardiovascular risk factors, and cardiovascular morbidity and mortality [1]. The most recent studies have also identified that men with CVD show significantly lower serum levels of bioavailable T than those men with negative coronary angiography (see Box 1 for CV safety issues and T-replacement). Numerous studies have found a direct association between male obesity and lower plasma T levels [2], and there is also an independent association between plasma $\mathrm{T}$ concentration and insulin sensitivity; [3,4] obese men with diabetes appear to be at particularly high risk of developing secondary hypogonadism [5]. This cluster of abnormalities is associated with an increased risk of diabetes and CVD, affecting not only quality of life but also life expectancy and the impact of resilience on surgical recovery and wound-healing. At present, biomarkers for resilience, which largely focuses on neuropeptide $\mathrm{Y}$, dehydroepiandrosterone and T [6] should be individualized for pre-operative management. As such, T represents one of the most prominent biomarkers, and its administration may represent a challenge for some categories of patients after surgery. Patient with acute myocardial infarction were found to have the lowest T levels ( $365.3 \mathrm{ng} / \mathrm{dL})$ and T level were also found to be independent predictors for CAD (odds ratio 0.311, 95\% confidence interval 0.174-0.512) [7]. Also, patients with CAD have lower $\mathrm{T}$ and oestradiol levels than healthy controls. These changes are inversely correlated with the degree of $\mathrm{CAD}$, suggesting that low plasma $\mathrm{T}$ levels may be involved in the increased risk of $\mathrm{CAD}$ in men [8]. In a pilot study, we found that in severely hypogonadal young men, acute administration of T-gel improves endothelial function suggesting that acute T supplementation could be a potential approach for both patients after surgery resilience or myocardial infarction; a short challenge of T could improve their vasodilatory responses to conventional treatment/s and recovery after surgery, when T plasma levels are severely blunted [9].

\section{Background on thyroid hormones}

The presence of non-thyroidal illness (NTI) in intensive care Unit (ICU) patients is frequent and the hypothesis that it could perhaps be maladaptive in this context has been repeatedly and continuously published over the last decades; however, the number of RCTs that address causality in association with adverse outcomes is surprisingly small. In fact, there are only four RTCs studying a total of 190 adult ICU patients 
that have addressed this question looking at clinically relevant outcomes. These studies were performed in four different study populations: patients with burn injuries, patients undergoing cardiac surgery, medical ICU patients, and patients suffering from acute kidney failure. Two of the RCTs used T4 and two used T3 as the hormone for treatment, with three out of four studies using a pharmacologic dose of thyroid hormones. All four studies showed that thyroid treatment further suppressed plasma TSH, and none showed a therapeutic benefit on patient outcome [10]. The combination of low cardiac output and elevated systemic vascular resistance i.e. after cardiopulmonary bypass has been investigated through thyroid supplementation and evaluation of cardiac hemodynamics in the perioperative period. Although improvements in cardiac output and systemic vascular resistance were observed, no significant changes in postoperative mortality were noted and thus thyroid supplementation is not routinely practiced in adult cardiac surgery. There was, however, evidence to suggest a role for postoperative T3 treatment to prevent postoperative atrial fibrillation. A recent study demonstrated that preoperative hypothyroidism was associated with postoperative atrial fibrillation in young-old patients thus suggesting that preoperative hypothyroidism maybe a useful biomarker for selecting those patients who most likely would benefit from preoperative thyroid supplementation in the prevention of postoperative atrial fibrillation [11]. Other authors have suggested that the presence of the NTI at admission is associated with an increased risk of postoperative myocardial dysfunction and death in patients undergoing coronary artery by-pass grafting [12]. It is clear that the name "NTI" during critical illness refers to a syndrome with different faces. Tolerating the early "fasting response' to critical illness and its concomitant changes in thyroid hormone parameters appears to be wise and beneficial. This applies to the NTI present in the majority of patients treated in ICU. However, the NTI that occurs in prolonged critically ill patients appears different in both its causes and consequences. Future studies should specifically target this selected population of prolonged critically ill patients, and, after excluding iatrogenic drug interferences, investigate the effect on outcome of treatment with hypothalamic releasing factors or thyroid hormones supplementation in adequately powered randomized controlled trials.

\section{Box 1 .}

\section{Cardiovascular safety issues and TD}

The relationship between TD and men's vascular health has a great impact because this is frequently associated with increased cardiovascular and all-cause mortality. Noteworthy, the occurrence of increased glucose, total cholesterol, low-density lipoprotein, proinflammatory cytokines, and myointimal carotid thickness may be associated with reduced T levels especially in cardiac older frail men. Screening for low T should be mandatory in high risk groups including those with type 2 diabetes, metabolic syndrome and obesity. Recent studies suggest warnings on T prescription in older frail men, but we regret that these studies had consistent bias in inclusion criteria and statistical evaluation. Data from studies conducted in more selected populations suggest that T replacement therapy may improve multiple surrogate markers of CVD as well as may reduce cardiovascular mortality. After analyzing the most important studies' limitation, we can conclude that at present there is insufficient evidence of a causal relationship between $\mathrm{T}$ therapy and adverse cardiovascular outcomes to support against T supplementation in older hypogonadal frail men.

\section{Evidence and proposal}

At present, there is no evidence that pre-operatory workup of the old-old male patient candidate to cardiac and extra-cardiac surgery should include total or free testosterone evaluation. However, clinical outcomes suggesting better surgical resilience in patients with higher endogenous plasma levels suggest its possible inclusion in the investigative routine workup. Similarly, evidence-based data on preoperative thyroid function in old-old patients predicting surgical outcomes are lacking. Undoubtedly, low-T3 levels before surgery in older patients are associated with major cardiac arrhythmias and this warrants deeper and wise evaluation workup in all patients undergoing surgery.

\section{References}

1. Corona G, Rastrelli G, Monami M, et al. Hypogonadism as a risk factor for cardiovascular mortality in men: a meta-analytic study, Eur. J. Endocrinol 2011;165:687-701.

2. Saad F, Gooren LJ. The role of testosterone in the etiology and treatment of obesity, the metabolic syndrome, and diabetes mellitus type 2. J Obes 2011;2011:pii 471584.

3. Kapoor D, Malkin CJ, Channer, KS, et al. Androgens, insulin resistance and vascular disease in men. Clin Endocrinol 2005;63: 239-50.

4. Srikanthan P, Karlamangla AS. Relative muscle mass is inversely associated with insulin resistance and prediabetes. Findings from the third national health and nutrition examination survey. J Clin Endocrinol Metab 2011;96:2898-03.

5. Traish AM, Saad F, Guay A. The dark side of testosterone deficiency: II. Type 2 diabetes and insulin resistance. J Androl 2009; $30: 23-32$.

6. Graham D, Becerril-Martinez G. Surgical resilience: a review of resilience biomarkers and surgical recovery. Surgeon 2014;12: 334-44.

7. Hu X, Rui L, Zhu T, et al. Low testosterone level in middle-aged male patients with coronary artery disease. Eur J Intern Med 2011; 22:e133-6.

8. Rosano GM, Sheiban I, Massaro R, et al. Low testosterone levels are associated with coronary artery disease in male patients with angina. Int J Impot Res. 2007;19:176-82.

9. Francomano D, Fattorini G, Gianfrilli D, et al. cute endothelial response to testosterone gel administration in men with severe hypogonadism and its relationship to androgen receptor polymorphism: a pilot study. J Endocrinol Invest 2016;39:265-71.

10. Klemperer JD, Klein I, Gomez M, et al. Thyroid hormone treatment after coronary artery bypass surgery. N Engl J Med 1995;333: 1522-7.

11. Worku B, Tortolani AJ, Gulkarov I, et al. Preoperative hypothyroidism is a risk factor for postoperative atrial fibrillation in cardiac surgical patients. J Card Surg 2015;30:307-12.

12. Cerillo AG, Storti S, Kallushi E, et al. The low triiodothyronine syndrome: a strong predictor of low cardiac output and death in patients undergoing coronary artery bypass grafting. Ann Thorac Surg 2014;97:2089-95. 